Journal of Fundamental and Applied Sciences

ISSN 1112-9867

Available online at

http://www.jfas.info

\title{
FACTORS LIVESTOCK AND MILKING ASSOCIATED WITH RISK OF MASTITIS IN CATTLE DAIRY FARMS IN ABOVEGROUND OF THE TUNISIA COASTAL SEMI-ARID
}

\author{
Y. M'Sadak*, L. Mighri, R. Haj Mbarek and K. Kraiem \\ Institut Supérieur Agronomique de Chott-Mariem, Université de Sousse, BP 47, 4042 Tunisie
}

Received: 11 April 2013 / Accepted: 15 November 2013 / Published online: 31 December 2013

\begin{abstract}
This study was carried out in the Sahel of Tunisia (Monastir region) on a sample covering 40 cattle herd's type above ground. We deduced the mastitis risk factors during the survey during visits for breeding and milking as well as from morphological and hygienic cow's assessments. The data's descriptive analysis has highlighted some significant effect factors on cellular levels and the risk of developing mastitis. For individual cell count (ICC), the significant effect factors $(\mathrm{P}<0.05)$ are: free stall, milking machine cleaning with only water, the poor condition of the piping, not elimination of the first milk jets and bad cleanliness of udders and the back legs of cows, whereas the herd cell count (HCC), the significant factors $(\mathrm{P}<0.05)$ are: cleaning the milking machine with water only, the frequency pulse $(\mathrm{FP})$ is too high or low.
\end{abstract}

Keywords: Dairy cows, Breeding and milking conditions; Somatic cell counts, Breast infections, Risk factors.

\section{INTRODUCTION}

Les mammites constituent généralement le trouble sanitaire le plus fréquent. Ces infections sont liées aux plus fortes répercussions économiques au sein de l'élevage bovin laitier $[7 ; 21$; $30 ; 35]$.

Author Correspondence, e-mail: msadak.youssef@yahoo.fr

Tel.: 0021673327 546; fax: 0021673327591.

ICID: 1078167 
L'infection peut être directement identifiée par la présence de germes pathogènes dans le lait [35]. Cependant les pathogènes mineurs sont associés à une augmentation du dénombrement cellulaire du lait [35]. Une telle infection a de multiples origines et est souvent de type subclinique, rendant le contrôle et la surveillance difficiles [8].

A l'heure où la gestion des coûts d'exploitation s'avère de plus en plus importante et doit être envisagée de façon plus systématique, le concept de facteur de risque d'apparition d'une mammite est devenu incontournable dans une approche préventive et curative de cette pathologie. Une telle démarche constitue la raison d'être du présent travail. Pour ce faire, des investigations ont été menées chez des petits troupeaux hors sol inscrits au contrôle laitier, conduits en milieu littoral semi-aride Tunisien.

L'objectif recherché était d'identifier quelques facteurs de risque des mammites bovines en rapport avec les comptages cellulaires individuels et collectifs. Ces facteurs ont été notamment explorés à l'échelle de l'élevage (logement, pratiques de traitement au tarissement, technique et équipement de traite) et à l'échelle de l'animal (conformation et propreté des mamelles) en milieu littoral semi-aride (Tunisie).

\section{MATÉRIEL ET MÉTHODES}

La présente étude a été réalisée dans la région de Monastir (zone littorale relevant du Sahel Tunisien) sur un échantillon de 40 petites unités bovines laitières. Tous les troupeaux considérés sont affiliés au contrôle laitier. Ils sont de faible taille variant de 2 à 29 vaches présentes (VP) et de 2 à 21 vaches en lactation (VL) avec en moyenne respectivement $9 \mathrm{VP}$ et 8 VL. On s'est intéressé au total de 364 VP dont 317 VL. Toutes les vaches sont de la même race (Frisonne Holstein) et elles sont, en majorité, soumises à la traite biquotidienne mécanique en pot (37 parmi les 40 élevages considérés). Le système d'élevage hors sol est caractéristique du site d'étude. Il se caractérise par l'insuffisance, voire l'absence des ressources fourragères à cause des ressources en eau généralement limitées tant quantitativement (zone semi-aride) que qualitativement (salinité excessive).

Chaque élevage a bénéficié d'au moins deux visites, l'une pour la traite matinale et l'autre pour celle de l'après-midi. Lors de ces visites, on a recueilli les informations relatives aux conditions d'élevage et de traite des vaches. Les fiches d'appréciation de la conformation des mamelles et de la propreté des vaches proposées par [37] ont été utilisées pour la notification des observations accomplies. 
Le prélèvement des échantillons individuels s'effectue après la traite complète de chaque vache alors que les échantillons collectifs sont prélevés à partir du lait de mélange de toutes les vaches de chaque troupeau, tout en agitant le lait pour avoir un échantillon représentatif. Les analyses cellulaires des échantillons laitiers prélevés ont été réalisées au sein du Laboratoire du Service de Contrôle Laitier du Centre d'Amélioration Génétique de Sidi Thabet (Tunisie), moyennant un compteur cellulaire automatique de type Fossomatic 4000 [20] mis au point par la Société Danoise Foss Electric, donnant les résultats du comptage cellulaire selon la méthode fluoro-opto-électronique qui constitue un moyen direct du comptage des cellules somatiques [15].

Pour l'étude des effets des facteurs de risque sur les comptages cellulaires individuels (CCI) et les taux cellulaires de troupeau (TCT), on a utilisé la procédure GLM du logiciel SAS (2000). Pour chaque facteur, on a comparé les moyennes de ses niveaux par le t-test (seuil $5 \%$ ) selon le modèle statistique $: \mathbf{Y i}=\mathbf{+} \mathbf{N F i}+\mathbf{e i}$

Avec : Yi $=$ Numération cellulaire, $\boldsymbol{\mu}=$ Moyenne, $\mathbf{N F}_{\mathbf{i}}=$ Effet du niveau du facteur de risque, $\mathbf{e}_{\mathbf{i}}=$ Erreur résiduelle.

\section{RÉSULTATS ET DISCUSSION}

\subsection{Contexte général de l'étude}

\subsubsection{Conditions d'élevage des vaches}

Tableau 1. Principales conditions d'élevage examinées chez les troupeaux suivis

\begin{tabular}{llcc}
\hline Variables & & Nombre Troupeaux & $\%$ \\
\hline \multirow{2}{*}{ Type Stabulation } & Entravée & 26 & 65 \\
& Libre & 14 & 35 \\
\hline \multirow{2}{*}{ Propreté Aire Couchage } & Propre & 14 & 35 \\
& Non Propre & 26 & 65 \\
\hline \multirow{2}{*}{ Propreté Lieu Traite } & Propre & 19 & 47 \\
& Non Propre & 21 & 53 \\
\hline \multirow{2}{*}{ Tarissement par Traitement antibiotique } & Pratiqué & 17 & 43 \\
& Absent & 23 & 57 \\
\hline
\end{tabular}

Le tableau 1 relate les principales conditions d'élevage considérées pour les exploitations enquêtées. La stabulation est libre chez $35 \%$ des exploitations. L'aire de couchage est non propre chez $65 \%$ des étables (humide et non paillée). La propreté du lieu de traite est mauvaise chez $53 \%$ des élevages. 
Le traitement antibiotique au tarissement des vaches laitières est un des fondements des programmes de maîtrise des infections mammaires [33]. Cependant, seulement $43 \%$ des éleveurs réalisent un traitement antibiotique au tarissement des vaches. Une étude relativement plus ancienne, réalisée en France par [25] a montré que le traitement au tarissement n'est adopté que par $38 \%$ des éleveurs.

\subsection{Conditions de traite des vaches}

\subsubsection{Caractéristiques des machines à traire}

Tableau 2. Caractéristiques des équipements de traite des vaches

\begin{tabular}{llll}
\hline Equipements de traite & & Effectif & \% \\
\hline \multirow{2}{*}{ Fréquence Pulsation } & $<55$ puls. /mn & 4 & 11 \\
& $55-60$ puls. /mn & 11 & 30 \\
& $>60$ puls. /mn & 22 & 59 \\
\hline \multirow{2}{*}{ Nettoyage Machine à traire } & Eau seulement & 7 & 19 \\
& Eau + javel & 27 & 73 \\
& Eau+ produit nettoyant & 3 & 8 \\
\hline \multirow{3}{*}{ Propreté Machine à traire } & Médiocre & 9 & 24 \\
& Moyenne & 13 & 36 \\
& Satisfaisante & 12 & 32 \\
& Bonne & 3 & 8 \\
\hline \multirow{2}{*}{ Etat Tuyauterie } & Médiocre & 10 & 27 \\
& Moyen & 15 & 41 \\
& Satisfaisant & 9 & 24 \\
& Bon & 3 & 8 \\
\hline
\end{tabular}

Le tableau 2 illustre les principales caractéristiques des machines à traire relevées au cours des visites réalisées. La fréquence de pulsation (FP) est le nombre de cycles effectués par le pulsateur de la machine à traire par mn. Elle doit être de l'ordre de 55 à 60 puls. /mn. [25] considère que l'apparition de nouvelles infections mammaires est liée indirectement avec la FP se situant à un niveau inférieur à 50 puls. /mn. Pour éviter une traite traumatisante, la FP ne doit pas dépasser 60 puls. /mn [4]. Dans l'étude courante, la FP comptée est supérieure à 60 puls. /mn chez 22 machines à traire $(59 \%)$. Ce résultat s'explique par le fait que les éleveurs ont tendance à augmenter la FP pour diminuer la durée de traite, ce qui engendre une répercussion néfaste sur la santé mammaire des vaches. 
$8 \%$ des éleveurs utilisent un produit nettoyant avec l'eau pour le nettoyage de la machine à traire. Malgré que la majorité des éleveurs utilise la solution d'eau additionnée de javel, cette dernière ne répond pas aux normes conventionnelles de nettoyage. Elle est à l'origine du mauvais état de propreté des équipements de traite chez 25 exploitations (68\%). L'état de la tuyauterie est satisfaisant à bon chez seulement 15 machines (40\%), en contre partie, il est médiocre chez 9 machines à traire $(24 \%)$.

Certains auteurs $[13 ; 18 ; 28]$ ont rapporté que le contrôle annuel des machines à traire a une influence sur les comptages cellulaires du lait par le fait qu'il permet de corriger les paramètres de fonctionnement des machines à traire, tout en respectant les normes et en traumatisant le moins possible les trayons. Cela se traduit par une baisse de la fréquence des mammites et une moindre numération cellulaire du lait.

\subsection{Pratiques de traite des vaches}

Les principales pratiques de traite relevées lors des suivis des chantiers de traite sont relatées dans le tableau 3.

Tableau 3. Caractéristiques des pratiques de traite des vaches

\begin{tabular}{|c|c|c|c|}
\hline Pratiques de traite & & Effectif & $\%$ \\
\hline \multirow{2}{*}{ Ordre Traite } & Sans & 37 & 93 \\
\hline & Avec & 3 & 7 \\
\hline \multirow{3}{*}{ Lavage Trayons } & Eau seulement & 3 & 8 \\
\hline & Eau + Lavette & 26 & 65 \\
\hline & Eau+Lavette+Savon & 11 & 27 \\
\hline \multirow{2}{*}{ Essuyage Trayons } & Sans & 35 & 88 \\
\hline & Avec & 5 & 12 \\
\hline \multirow{3}{*}{ Elimination $1^{\text {ers }}$ jets } & Sans & 27 & 68 \\
\hline & Sur sol & 6 & 15 \\
\hline & Dans récipient & 7 & 17 \\
\hline \multirow{4}{*}{ Type Egouttage } & Sans & 3 & 8 \\
\hline & Machine & 26 & 70 \\
\hline & Manuel & 6 & 16 \\
\hline & Machine et Manuel & 2 & 6 \\
\hline \multirow{2}{*}{ Désinfection Trayons } & Pratiquée & 19 & 47 \\
\hline & Absente & 21 & 53 \\
\hline
\end{tabular}


Certains auteurs insistent sur l'importance hygiénique du nettoyage des trayons avant la traite. A ce propos, le lavage de la mamelle avant la traite a pour objectif de faire disparaître les souillures, de favoriser la descente du lait et de détruire les germes. Le lavage doit être obligatoirement suivi d'un essuyage des quartiers lavés. Laver sans essuyer est pire que de ne pas laver $[12 ; 23]$.

$93 \%$ des trayeurs ne respectent pas l'ordre de traite. Ce résultat observé est élevé par rapport à celui trouvé par [26]. La totalité des élevages pratique le lavage des trayons avant la traite. Plus que la moitié (65\%) lavent les trayons avec une lavette collective, $27 \%$ des trayeurs utilisent du savon avec la lavette mouillée pour améliorer la qualité du nettoyage des trayons. Malgré son importance, l'essuyage des trayons n'est pratiqué que par $12 \%$ des trayeurs. Ce résultat est proche de celui trouvé par [26] qui ont relevé un mauvais essuyage chez $83 \%$ des élevages. Une autre étude réalisée par [27] dans la région de Mahdia (berceau laitier de la Tunisie), appartenant également au Sahel Tunisien, a rapporté un résultat peu différent de celui relevé lors de cette étude. En effet, $33 \%$ des éleveurs considérés ne pratiquent pas l'essuyage.

L'élimination des premiers jets est une opération qui permet, d'une part, d'éliminer le lait particulièrement riche en germes, se trouvant directement au-dessus du canal du trayon et dans la citerne du trayon, et d'autre part, de contrôler la qualité du lait, en vérifiant la présence de signes cliniques de mammite. $[10 ; 16 ; 17 ; 28]$ rapportent que l'absence de l'élimination des premiers jets avant la mise en place des gobelets-trayeurs représente un facteur de risque des mammites subcliniques. En effet, les premiers jets de lait sont les plus riches en germes et leur élimination les empêche de passer dans la machine à traire, et par conséquent, réduit les contaminations ultérieures des mamelles par la machine. L'élimination des premiers jets avant la traite est adoptée par seulement $32 \%$ des trayeurs. Dans une étude relativement ancienne élaborée par [11], cette pratique est absente chez environ $89 \%$ des éleveurs, et dans une autre étude plus récente, accomplie par [26], l'élimination des premiers jets n'est réalisée que par $33 \%$ des trayeurs.

Pour obtenir le lait d'égouttage mécanique, l'éleveur est alors tenté de pratiquer la surtraite. Cette pratique est de toute manière vouée à l'échec, car, outre le fait qu'elle aggrave les lésions mécaniques, elle favorise l'introduction de germes dans les quartiers [19]. Une entrée d'air par la griffe à lait occasionnée lors de l'égouttage, inverse fortement les pressions et provoque le phénomène d'impact. Par ailleurs, c'est en fin de traite que les conséquences du phénomène d'impact sont les plus néfastes, car si des germes pénètrent dans le trayon, le débit 
de lait est trop faible pour les chasser [24]. La majorité des trayeurs (92\%) adopte l'égouttage (particulièrement, l'égouttage mécanique).

[28] rapportent que les élevages qui pratiquent la désinfection des trayons ont des taux cellulaires inférieurs à ceux qui ne la pratiquent pas. Cependant, la désinfection des trayons après la traite n'est opérée que chez $47 \%$ des trayeurs. Ce résultat est proche de celui annoncé par [26].

\subsection{Recherche de l'impact des facteurs de risque sur les numérations cellulaires}

\subsubsection{Relations entre les conditions d'élevage et les CCI}

Tableau 4. Impact des conditions d'élevage sur les CCI (x1000 cell. /ml)

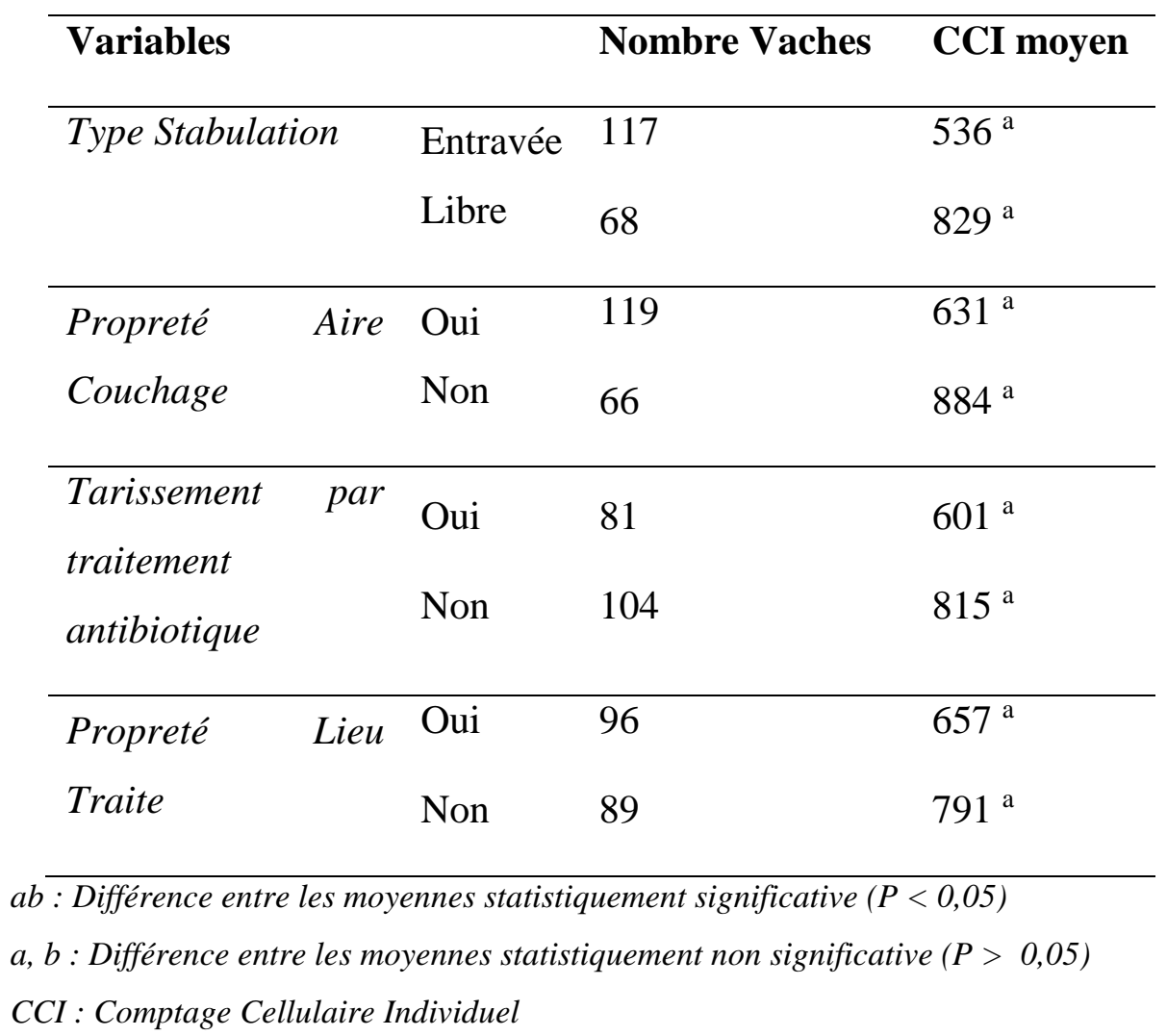

Les pratiques d'élevage considérées dans cette étude n'ont pas montré d'influence sur les CCI des vaches (Tableau 4) malgré que plusieurs études ont témoigné qu'une hygiène insuffisante de l'environnement global des bâtiments d'élevage et des aires de repos est fortement liée à des CCI élevés.

La stabulation libre a montré un CCI moyen plus élevé (829000 cell. /ml) que celui repéré avec la stabulation entravée (536000 cell. $/ \mathrm{ml}$ ) avec une tendance d'influence du type de stabulation sur les CCI $(\mathrm{P}<0,10)$. 
L'aire de couchage a présenté une légère différence entre les CCI moyens des aires propres et des aires non propres avec un avantage pour les aires propres, toutefois, l'analyse statistique a révélé que cette différence est non significative (Tableau 4).

La période de tarissement est une période critique pour la vache laitière. Malgré que la mamelle soit en repos, le risque d'apparition de nouvelles infections reste élevé. Le traitement antibiotique au tarissement est un des plans de maîtrise des infections intra-mammaires [32]. $44 \%$ des vaches ont subi le traitement antibiotique au tarissement. Ces vaches ont enregistré des CCI moyens légèrement plus faibles que ceux des vaches non traitées, cette différence n'est pas significative (Tableau 4).

Le lieu de traite des vaches est propre chez presque la moitié de l'échantillon (52 \%), la traite du reste des vaches se déroule dans un lieu non propre. L'analyse a dévoilé que la faible différence entre les CCI moyens des lieux de traite propres et ceux non propres est une différence non significative.

\section{Relations entre les conditions de traite et les CCI}

\subsection{Effet des équipements de traite sur les CCI}

Tableau 5. Quelques caractéristiques de la machine à traire et CCI (x1000 cell. /ml)

\begin{tabular}{llll}
\hline Variables & & $\begin{array}{l}\text { Nombre } \\
\text { Vaches }\end{array}$ & $\begin{array}{l}\text { CCI } \\
\text { moyen }\end{array}$ \\
\hline $\begin{array}{l}\text { Nettoyage } \\
\text { Machine à traire }\end{array}$ & Eau + produit nettoyant & 20 & $353^{\mathrm{a}}$ \\
& Eau+javel & 128 & $503^{\mathrm{a}}$ \\
\hline \multirow{2}{*}{$\begin{array}{l}\text { Etat } \\
\text { Tuyauteries }\end{array}$} & Bon & 14 & $1303^{\mathrm{b}}$ \\
& Satisfaisant & 12 & $470^{\mathrm{a}}$ \\
\hline \multirow{2}{*}{$\begin{array}{l}\text { Fréquence } \\
\text { Pulsation }\end{array}$} & Médiocre & 68 & $504^{\mathrm{a}}$ \\
\multicolumn{2}{c}{ ab : Différence entre les moyennes statistiquement significative $(P<0,05)$} & $665^{\mathrm{ab}}$ \\
$\begin{array}{l}\text { a, } b \text { : Différence entre les moyennes statistiquement non significative }(P>0,05) \\
\text { CCI : Comptage Cellulaire Individuel }\end{array}$ & 32 & $967^{\mathrm{b}}$ \\
\hline
\end{tabular}

L'étude statistique a montré que les caractéristiques de la machine à traire ont une incidence sur les CCI (Tableau 5). La machine à traire doit être nettoyée après chaque usage. Une machine à traire propre est indispensable pour conserver la qualité cellulaire du lait jusqu'à sa 
consommation. Il est donc recommandé d'utiliser deux types de solution par alternance (alcaline / acide) et régulièrement [38]. L'ignorance de ces recommandations explique la relation entre le nettoyage de la machine à traire avec l'eau seulement et les CCI élevés $(\mathrm{P}<$ 0,05). De même, l'état médiocre de la tuyauterie a un impact sur les CCI $(\mathrm{P}<0,05)$.

Pour la fréquence de pulsation (FP), on a enregistré les CCI les plus élevés avec les fréquences supérieures à 60 puls. /mn, ce résultat est conforme avec celui aperçu par [26]. Des FP trop élevées entrainent des lésions aux extrémités des trayons alors que des FP trop faibles augmentent le temps de traite inutilement [14].

\subsection{Effet des pratiques de traite sur les CCI}

Tableau 6. Effet des pratiques de traite des CCI (x1000 cell. /ml)

\begin{tabular}{|c|c|c|c|}
\hline Variables & & Nombre Vaches & CCI moyen \\
\hline \multirow{2}{*}{ Respect Ordre Traite } & Oui & 9 & $389^{a}$ \\
\hline & Non & 176 & $738^{a}$ \\
\hline \multirow{3}{*}{ Lavage Trayons } & Lavage + Essuyage & 22 & $675^{a}$ \\
\hline & Lavage sans essuyage & 128 & $693^{\mathrm{a}}$ \\
\hline & Sans lavage ni essuyage & 35 & $701^{\mathrm{a}}$ \\
\hline \multirow{3}{*}{ Elimination $1^{\text {ers }}$ jets } & Récipient & 26 & $376^{\mathrm{a}}$ \\
\hline & Sur Sol & 33 & $567^{a b}$ \\
\hline & Non & 126 & $843^{b}$ \\
\hline \multirow{2}{*}{ Désinfection Trayons } & Oui & 107 & $707^{\mathrm{a}}$ \\
\hline & Non & 78 & $740^{a}$ \\
\hline \multirow{2}{*}{ Hygiène Traite } & Oui & 68 & $520^{a}$ \\
\hline & Non & 117 & $838^{\mathrm{a}}$ \\
\hline \multirow{2}{*}{ Egouttage Mamelle } & Oui & 115 & $693^{\mathrm{a}}$ \\
\hline & Non & 70 & $738^{a}$ \\
\hline
\end{tabular}

Les pratiques de traite en majorité n'ont pas d'incidence sur les CCI (Tableau 6), sauf pour l'élimination des premiers jets. Les CCI sont plus élevés avec la non élimination des premiers jets $(\mathrm{P}<0,05)$. Ce résultat est similaire à celui trouvé par [6]. Plusieurs études ont montré que la non élimination des premiers peut être un des facteurs responsables de l'élévation des CCI [28; $31 ; 34]$. Le lavage des mamelles avec des lavettes individuelles ou avec des douchettes 
à jet dirigé, suivi d'un essuyage rigoureux sont des mesures d'hygiène recommandées par [4].

Ces mesures ont révélé leur efficacité pour la réduction des numérations cellulaires du lait.

\section{Effet des caractéristiques mammaires et de la propreté des vaches sur les CCI}

\subsection{Conformation des mamelles et CCI}

L'évaluation de la conformation des mamelles a révélé que la profondeur du pis est conforme chez $62 \%$ des vaches, les autres caractéristiques qui sont la qualité des attaches, la position, la taille et la forme des trayons sont conformes chez environ la moitié des vaches (Tableau 7). Dans cette étude, aucune association significative n'a été observée entre conformation des mamelles et CCI. Toutefois, on constate que les CCI sont plus élevés avec les caractéristiques non conformes (Tableau 7). Par contre, d'après une étude réalisée par [22], $17 \%$ des vaches considérées, ayant la position des trayons au dessous du jarret ont contribué à $24 \%$ des cas des mammites enregistrées.

Tableau 7. Relation entre la conformation des mamelles et les CCI (x1000 cell. /ml)

\begin{tabular}{|c|c|c|c|}
\hline Variables & & $\begin{array}{l}\text { Nombre } \\
\text { Vaches }\end{array}$ & CCI moyen \\
\hline Profondeur & Conforme & 114 & $760^{a}$ \\
\hline Mamelle & Non & 71 & $778^{\mathrm{a}}$ \\
\hline Qualité & Conforme & 88 & $700^{a}$ \\
\hline Attaches & Non & 97 & $835^{\mathrm{a}}$ \\
\hline Position & Conforme & 93 & $750^{a}$ \\
\hline Trayons & Non & 92 & $792^{\mathrm{a}}$ \\
\hline Forme & Conforme & 92 & $741^{a}$ \\
\hline Trayons & Non & 93 & $801^{\mathrm{a}}$ \\
\hline
\end{tabular}

\subsection{Propreté des vaches et CCI}

L'évaluation de la propreté des vaches a été accomplie par l'appréciation de la propreté des mamelles (pis) et des pattes arrière ainsi que celle des flancs et des cuisses avec trois niveaux de propreté (bonne, moyenne et mauvaise) et les résultats sont relatés dans le tableau 8. 
La propreté du pis était bonne chez $30 \%$ des vaches, alors que la propreté des flancs et cuisses était bonne chez seulement $9 \%$ des vaches. Ces deux paramètres d'appréciation sont des indicateurs d'hygiène de l'aire de couchage et de la litière. La propreté des pattes arrière était bonne chez $20 \%$ des vaches, c'est un indicateur de l'hygiène des surfaces de marche et d'exercice. La situation actuelle mérite une intervention rapide, en vue d'améliorer l'entretien des étables pour une meilleure propreté des vaches.

L'étude de l'effet de la propreté des vaches sur les CCI est relatée dans le tableau 8. Elle a dévoilé qu'une propreté mauvaise du pis est associée à un CCI moyen de 1423000 cell. /ml, qui est très élevé par rapport à celui des propretés bonne et moyenne $(\mathrm{P}<0,05)$. La propreté des pattes arrière a une influence sur les CCI $(\mathrm{P}<0,05)$, alors que l'on a relevé une tendance pour l'association propreté des flancs et cuisses et CCI $(\mathrm{P}<0,10)$.

Tableau 8. Relation entre la propreté des mamelles et les CCI (x1000 cell. /ml)

\begin{tabular}{|c|c|c|c|}
\hline Variables & & $\begin{array}{l}\text { Nombre } \\
\text { Vaches }\end{array}$ & CCI moyen \\
\hline \multirow{3}{*}{ Mamelle } & Bonne & 56 & $636^{\mathrm{a}}$ \\
\hline & Moyenne & 101 & $688^{a}$ \\
\hline & Mauvaise & 28 & $1423^{b}$ \\
\hline \multirow{3}{*}{ Pattes Arrières } & Bonne & 37 & $587^{\mathrm{a}}$ \\
\hline & Moyenne & 109 & $837^{a b}$ \\
\hline & Mauvaise & 39 & $1223^{b}$ \\
\hline \multirow{3}{*}{$\begin{array}{l}\text { Flancs } \\
\text { Cuisses }\end{array}$} & Bonne & 17 & $433^{a}$ \\
\hline & Moyenne & 113 & $681^{\mathrm{a}}$ \\
\hline & Mauvaise & 55 & $1061^{a}$ \\
\hline
\end{tabular}




\section{Relation des conditions d'élevage et de traite avec les TCT}

\subsection{Conditions d'élevage et TCT}

Tableau 9. Relation de quelques caractéristiques d'élevage avec les TCT (x1000 cell. /ml)

\begin{tabular}{llll}
\hline Variables & & $\begin{array}{l}\text { Nombre } \\
\text { Troupeaux }\end{array}$ & TCT moyen \\
\hline Type Stabulation & Entravée & 25 & $721^{\mathrm{a}}$ \\
& Libre & 15 & $818^{\mathrm{a}}$ \\
\hline Propreté Aire Couchage & Oui & 26 & $733^{\mathrm{a}}$ \\
& Non & 14 & $807^{\mathrm{a}}$ \\
\hline Tarissement par & Oui & 17 & $739^{\mathrm{a}}$ \\
traitement antibiotique & Non & 23 & $813^{\mathrm{a}}$ \\
\hline \multirow{2}{*}{ Propreté Lieu Traite } & Oui & 19 & $721^{\mathrm{a}}$ \\
& Non & 21 & $836^{\mathrm{a}}$ \\
\hline
\end{tabular}

$a b$ : Différence entre les moyennes statistiquement significative $(P<0,05)$

$a, b:$ Différence entre les moyennes statistiquement non significative $(P>0,05)$

TCT : Taux Cellulaire de Troupeau

La comparaison des moyennes des classes des variables, relative aux pratiques d'élevage, associées à des TCT élevés figure dans le tableau 9. En effet, les caractéristiques d'élevage choisies lors de cette étude n'ont pas montré d'influence sur les numérations cellulaires du lait $(\mathrm{P}>0,05)$.

L'influence du système de stabulation sur l'importance des teneurs en cellules somatiques est encore contestée, mais elle fait l'objet d'études. Une litière sèche et propre peut diminuer la propagation des agents infectieux. L'analyse des TCT a révélé que, malgré qu'il n' y a pas de différence significative, les taux les plus élevés sont liés à la stabulation libre.

Après l'analyse, il s'est avéré que la différence n'est pas significative entre l'application du traitement au tarissement et son absence. Les TCT sont légèrement plus faibles pour les troupeaux dont les vaches sont traitées par des antibiotiques lors du tarissement que chez les troupeaux qui n'utilisent pas d'antibiotiques (Tableau 9).

\subsection{Conditions de traite et TCT}

L'influence des principales caractéristiques de la machine à traire sur les TCT est relatée dans le tableau 10. 
Tableau 10. Effet des caractéristiques de la machine à traire sur les TCT(x1000 cell. /ml)

\begin{tabular}{|c|c|c|c|}
\hline Variables & & $\begin{array}{l}\text { Nombre } \\
\text { Troupeaux }\end{array}$ & $\begin{array}{l}\text { TCT } \\
\text { moyen }\end{array}$ \\
\hline Nettoyage & Eau+ produit nettoyant & 2 & $239^{a}$ \\
\hline Machine & Eau+javel & 28 & $838^{a b}$ \\
\hline à traire & Eau seulement & 7 & $1076^{b}$ \\
\hline \multirow{4}{*}{$\begin{array}{l}\text { Etat } \\
\text { Tuyauteries }\end{array}$} & Bon & 3 & $655^{\mathrm{a}}$ \\
\hline & Satisfaisant & 12 & $791^{a}$ \\
\hline & Moyen & 13 & $861^{a}$ \\
\hline & Médiocre & 9 & $1234^{\mathrm{a}}$ \\
\hline Fréquence & $<55$ puls. $/ \mathrm{mn}$ & 4 & $845^{a b}$ \\
\hline \multirow[t]{2}{*}{ Pulsation } & $55-60$ puls. $/ \mathrm{mn}$ & 5 & $652^{a}$ \\
\hline & $>60$ puls. $/ \mathrm{mn}$ & 28 & $1030^{b}$ \\
\hline
\end{tabular}

Le nettoyage des machines à traire a une incidence sur les TCT $(\mathrm{P}<0,05)$. En effet, les TCT moyens des élevages qui font le nettoyage des machines avec un produit nettoyant sont plus faibles que ceux des élevages qui font le nettoyage de leurs machines avec l'eau seulement. En termes de relation entre le nettoyage de la machine à traire et les taux cellulaires, ce résultat est proche de celui trouvé par [4] et [5].

L'état des tuyauteries ne présente pas une influence sur les TCT $(\mathrm{P}>0,05)$. Un tel résultat est différent de celui trouvé par [4], qui a annoncé que les manchons-trayeurs usagés ayant une surface rugueuse et craquelée sont des sources très importantes et chroniques de contamination. [18], [13] et [28] ont rapporté que le contrôle annuel des machines à traire a une influence sur les TCT des élevages par le fait que le contrôle annuel de la machine permet de corriger les paramètres de fonctionnement afin qu'ils respectent les normes et traumatisent le moins possible les trayons. A ce propos, il convient de rappeler que l'effet se traduit par une baisse de la fréquence des mammites et une moindre numération cellulaire du lait.

La pulsation est l'un des facteurs principaux de la machine à traire impliqués dans le développement des infections mammaires. L'étude de la relation entre la Fréquence de Pulsation (FP) et les taux cellulaires a montré que les FP > 60 puls/mn sont liées à des TCT élevés $(\mathrm{P}<0,05)$. [4] ont annoncé que la pulsation n'est pas en relation directe avec 
l'apparition de nouvelles infections, mais l'absence ou la quasi-absence de pulsation est à l'origine d'une augmentation sensible des infections mammaires. Dans tous les cas, il faut éviter l'emploi d'une pulsation rapide ou faible. Le respect d'une pulsation de 55 à 60 puls. /mn pour traire les vaches est une condition nécessaire, mais non suffisante, car, il faut également respecter les normes pour les autres paramètres de fonctionnement du pulsateur : rapport de pulsation, rapport de décalage, ...

Tableau 11. Relation de quelques pratiques de traite avec les TCT (x1000 cell. /ml)

\begin{tabular}{|c|c|c|c|}
\hline Variables & & $\begin{array}{l}\text { Nombre } \\
\text { Troupeaux }\end{array}$ & TCT moyen \\
\hline \multirow{2}{*}{$\begin{array}{l}\text { Respect } \\
\text { Traite }\end{array}$} & Oui & 3 & $746^{\mathrm{a}}$ \\
\hline & Non & 37 & $1216^{\mathrm{a}}$ \\
\hline \multirow{3}{*}{ Lavage Trayons } & Lavette+savon & 11 & $774^{\mathrm{a}}$ \\
\hline & Lavette+eau & 26 & $777^{\mathrm{a}}$ \\
\hline & Eau seulement & 3 & $801^{\mathrm{a}}$ \\
\hline \multirow{2}{*}{ Essuyage Trayons } & Oui & 5 & $775^{a}$ \\
\hline & Non & 35 & $827^{\mathrm{a}}$ \\
\hline \multirow{3}{*}{ Elimination $1^{\text {ers }}$ jets } & Récipient & 7 & $674^{\mathrm{a}}$ \\
\hline & Sur & 6 & $786^{a}$ \\
\hline & Non & 27 & $888^{\mathrm{a}}$ \\
\hline \multirow{2}{*}{$\begin{array}{l}\text { Désinfection } \\
\text { Trayons }\end{array}$} & Oui & 19 & $692^{a}$ \\
\hline & Non & 21 & $881^{\mathrm{a}}$ \\
\hline \multirow{2}{*}{ Hygiène Traite } & Oui & 13 & $697^{\mathrm{a}}$ \\
\hline & Non & 27 & $822^{a}$ \\
\hline \multirow{2}{*}{ Egouttage Mamelle } & Oui & 26 & $728^{a}$ \\
\hline & Non & 14 & $881^{\mathrm{a}}$ \\
\hline \multicolumn{4}{|c|}{$a b:$ Différence entre les moyennes statistiquement significative $(P<0,05)$} \\
\hline \multicolumn{4}{|c|}{$\begin{array}{l}a, b: \text { Différence entre les moyennes statistiquement non significative }(P>0,05) \\
C C I: \text { Comptage Cellulaire Individuel }\end{array}$} \\
\hline
\end{tabular}

Pour les pratiques de traite suivies dans cette étude, on n'a pas enregistré d'influence remarquable sur les TCT (Tableau 11). L'ordre de traite a une tendance d'influence sur les TCT $(\mathrm{P}<0,10)$. Le lavage des trayons dans cette étude ne présente pas une influence sur les 
TCT $(\mathrm{P}>0,05)$. L'élimination des premiers jets a une tendance d'influence sur les TCT $(\mathrm{P}<$ 0,10). Les premiers jets de lait sont plus riches en germes et leur élimination évite la contamination du lait de mélange. Quelques études ont montré que la non élimination des premiers jets peut être un des facteurs responsables de l'élévation des TCT [28; $31 ; 34]$. L'égouttage des mamelles après la traite ne peut pas être considéré comme un facteur de risque des mammites ou un facteur de variation des TCT à partir des résultats obtenus lors de ce travail $(\mathrm{P}>0,05)$. Le résultat acquis concorde avec celui trouvé par [28], mais il est différent de celui trouvé par [23].

\section{CONCLUSION}

Cette étude a constitué une première approche de la description des facteurs de risque des mammites bovines. Elle a permis de mettre en évidence quelques constatations essentielles à travers la description des conditions d'élevage, les équipements et les pratiques de traite en rapport avec l'analyse des Comptages Cellulaires Individuels (CCI) et des Taux Cellulaires de Troupeau (TCT) dans de petites étables hors sol de la Tunisie littorale semi-aride.

La plupart des exploitations considérées a révélé un mauvais entretien du logement caractérisé par une mauvaise hygiène des vaches, un manque de contrôle de la machine à traire. Les règles d'hygiène et les bonnes pratiques de la traite des vaches ne sont pas respectées. Ces conditions expliquent les taux cellulaires élevés observés à la fois au niveau individuel et au niveau des troupeaux. Ces élévations cellulaires étaient le signe d'un état mammaire infecté.

Pour en réduire l'incidence et la prévalence, la mise en place de plans de lutte contre les mammites se justifie. Ces plans ne peuvent être qu'à certaines conditions impératives : Que l'éleveur ait compris toutes les défaillances pour chaque point et ne se limite pas à la simple compréhension. Que des objectifs à long terme soient fixés de façon à ce que l'éleveur ne soit pas déçu par un manque de résultats après quelques mois d'application. Que les objectifs soient réalistes et conformes à ce que l'on peut attendre du plan en n'oubliant pas qu'il s'agit d'un plan de prévention et non d'extraction.

Parallèlement, le développement d'un diagnostic et d'une assistance technique efficace des conditions générales (d'élevage, du fonctionnement des machines à traire, des pratiques de traite) semble fondamental.

Les visites des élevages, l'analyse des données de santé mammaire, les visites de traite et des bâtiments, l'apport des conseils nécessaires et le suivi régulier sont les étapes qui doivent être adoptées pour contribuer aux objectifs fixés. 


\section{REFERENCES BIBLIOGRAPHIQUES}

[1] Aouadi A., 1991: Contribution à l'étude des paramètres zootechniques dans les grands élevages bovins du gouvernorat de béja. Thèse méd. Vét., enmv sidi thabet, tunisie, 100 p.

[2] Barnouin J., Geromegnace N., Chassagne M., Dorr N., Sabatier P., 1999. Facteurs structurels de variation des niveaux de comptage cellulaire du lait et de fréquence des mammites cliniques dans 560 élevages bovins répartis dans 21 départements français. INRA Prod. Anim., 12 (1), 39-48.

[3] Ben Dhiab H., 2001. Etude des mammites dans les petits élevages bovins de la région de Monastir, PFE INA Tunis, 2001, Tunisie, 54-75.

[4] Billon P. Sauvee O., Menard J.L., Gaudin V., 1998. Influence de la traite et de la machine à traire sur les numérations cellulaires et les infections mammaires chez la vache laitière. Renc. Rech. Ruminants, 1998, 5, 305-312.

[5] Billon P., Gaudin V., 2008. Quels réglages pour quelle machine à traire ? Institut de l'Elevage et Chambre d'Agriculture de Loire Atlantique, France, 7p.

[6] Bony J., Contamin V., Metais J., Nabeneza S., Tillard E., Coulon J.B., Juanes X., 2004

- Principaux facteurs qui influencent la qualité sanitaire du lait à la Réunion, Renc. Rech. Rum., 11, p 116.

[7] Coulon J.B., Lescourret F., 1997. Effet des mammites cliniques sur la production chez la vache laitière. Renc. Rech. Ruminants, 4, 265-268.

[8] Delfosse C., Froidmont E, Curnel Y., Humblet M.F., Hanzen Ch., Bertozzi C., Bartiaux-Thill N., 2006. Etude écopathologique des facteurs de risque des mammites dans les élevages laitiers en Wallonie. Renc. Rech. Ruminants, 2006, 13, 440.

[9] Dohoo J.R., Lesile K.E. 1990. Evaluating of changes in somatic cell count as indicator of new intramammary infection. In: int. Symp. Bovine mastitis, national mastitis council, indianapolis, in, USA, 13-16, 320-325.

[10] Ewy A., 2003. Préparation à la traite chez la vache laitière: comparaison des différentes méthodes de nettoyage des trayons. Revue UFA, 1- 4.

[11] Fabre J.M., Bazin S., Faroult B., Cail P., Berthelot X., 1996. Lutte contre les mammites. Résultats d'enquête réalisée auprès de 1038 élevages français. Bulletin des GTV, (2), 13-16.

[12] Faroult B., 1990. Assistance à la traite et qualité du lait. Bull. G.T.V. 3, B, 353, 25-39 
[13] Faroult B., 1994. Méthodologie d'approche des infections mammaires en troupeau laitier et maîtrise de la qualité hygiénique du lait. Rec. Méd. Vét., 170 (6/7), 469-478.

[14] France Agricole, 2009. Traite des vaches laitières : matériel, installation, entretien. Institut de l'Elevage, 555 p.

[15] Gambo H. et Agnem-Etchike C., 2001. Dépistage de mammites subcliniques chez des vaches goudali en lactation au nord Cameroun, Revue Elev. Méd. Vét. Pays trop, 54 (1), 5-10. [16] Garland G. A., 1997. Technique de traite normale. Fiche technique du Ministère de l'agriculture et l'alimentation Ontario, Agdex , 410 -725.

[17] Gerald R., Bodman P.E., 1997. Do you practice good milking procedures? University of Nebraska - Lincoln, cooperative extension, Institute of Agriculture and Natural Resources.

[18] Goodger W.J., Farver T., Pelletier J., Johnson P., Desnayer G., Galland J., 1993. The association of milking management practices with bulk tank somatic cell counts. Prev. Vet. Med, 15, 235-251.

[19] Gourreau JM, Arfi L, Brouillet P, Coussi G. Fieni F, Lacombe JF, Paulizzi L, Simonin F. Radigue PE., 1995. Accidents et maladies du trayon. Ed. France Agricole, Paris, p 287.

[20] Grappin R., Jeunet R., 1974 : Premiers essais de l'appareil «Fossomatic» pour la détermination automatique du nombre de cellules du lait, Revue le lait, ${ }^{\circ}$ 539-540, 627-644.

[21] Guerin-Faublee V., Carret G., Houffschmitt P., 2003. In vitro activity of 10 agents against bacteria isolated from cows with clinical mastitis. The veterinary record, 466-471.

[22] Hanzen Ch., 2008 - Physiologie de la glande mammaire et du trayon de la vache laitière. Faculté de Médecine Vétérinaire Service de Thériogenologie des animaux de production. $49 \mathrm{p}$.

[23] Hutton C.T., Fox L.K., Hancock D.D., 1991. Risk factors associated with herd-group milk somatic cell count and prevalence of coagulase-positive staphylococcol intramammary infections. Prev. Vet. Med., 11, 25-35.

[24] Lacombe JF., 1995. Pathologie liée à la machine à traire : accidents et maladies du trayon. Edition France Agricole, 189-231.

[25] Mezine M., 2006. Analyse descriptive des facteurs de risque liés aux mammites dans des élevages d'une clientèle des Ardennes appliquant la démarche GTV partenaire. Thèse méd. Vét., ENMV Alfort, France, 146. 
[26] M'Sadak Y., Mighri L., Kraiem K., 2010. Effet des conditions de traite sur la santé mammaire des vaches laitières et estimation des pertes en lait consécutives dans la région de Mahdia en Tunisie. Revue élev. Méd. Vét. Pays trop., 63 (1-2), 35-39.

[27] M'Sadak Y., Mighri L., Kraiem K., 2011. Etude de la situation sanitaire mammaire à partir des taux cellulaires de troupeau et estimation des pertes laitières engendrées chez des ateliers bovins hors sol en Tunisie. Revue Nature et Technologie, $n^{\circ}$ 04/janvier 2011, 08 - 14. [28] Mtaallah B., Oubey Z., Hammami H.,2002. Estimation des pertes de production en lait et des facteurs de risque des mammites subcliniques à partir des numérations cellulaires de lait de tank en élevage bovin laitier. Rev. Méd. Vét., 2002, 153, 4, 251-260.

[29] Noireterre Ph., 2006. Suivi de comptages cellulaires et d'examens bactériologiques lors de mammites cliniques chez la vache laitière, Thèse Vétérinaire Lyon, France, 98 p.

[30] Poutrel B., 1985. Généralités sur les mammites de la vache laitière : Processus infectieux, épidémiologie, diagnostic, méthodes de contrôle. Rec. Méd. Vét., 161 (6-7), 497511.

[31] Rasmussen M.D., Galton D.M., Petersson L.G., 1991. Effects of premilking teat preparation on spores anaerobes, bacteria, and iodine residues in milk. J. Dairy sci., 74, $2472-$ 2478.

[32] Robert A., Roussel P., Bareille N., Ribaud D., Serieys F., Leguenic M., Baudet H., Heuchel V. et Seegers H., 2005. Facteurs de risque de nouvelles infections intra-mammaires pendant la période tarie chez des vaches laitières dans des troupeaux pratiquant le traitement sélectif au tarissement. Renc. Rech. Ruminants., 2005, 12, 263-266.

[33] Roussel Ph., Souday E., Le Guenic M., 2003. Enquête sur le traitement sélectif au tarissement dans 33 troupeaux de l'ouest de la France. Renc. Rech. Ruminants. 10. p 297.

[34] Schukken Y.H., Grommers F.J., Van De Geer D., Erb H.N., Brand A., 1991. Risk factors for clinical mastitis in herds with a low bulk milk somatic cell count; 2 risk factors for escherichia coli and staphylococcus aureus. J. Dairy sci., 74, 826-832.

[35] Seegers H., Menard J.L., Fourichon C., 1997. Mammites en élevage bovin laitier : importance actuelle, épidémiologie et plans de prévention. Renc. Rech. Ruminants, 4, 233242.

[36] Selze J.C., 1999. Taux cellulaires élevés, qui est responsable ? Mammites cliniques ou subcliniques ? Bull. Tech. Insémin. Artif., 91, 31-33.

[37] Simon D., Jean Philippe R., 2005. Guide vétérinaire d'investigation sur la santé de la glande mammaire. Réseau canadien de recherche sur la mammite bovine RCRMB, Faculté De Médecine Vétérinaire, Université De Montréal, Canada, j2s 7c6, 26 p. 
[38] Wattiaux M.A., 2005. Procédure de traite, Institut Babcock pour la Recherche et le Développement International du Secteur Laitier, Etats-Unis, 5 p.

\section{How to cite this article:}

M'Sadak Y. Mighri L. Haj Mbarek R. Kraiem K. Factors livestock and milking associated with risk of mastitis in cattle dairy farms in aboveground of the Tunisia coastal semi-arid. $\mathrm{J}$ Fundam. Appl Sci. 2013, 5(2), 137-155 